\title{
Literarische Transformationen sexueller Gewalt in der Hebräischen Bibel
}

\author{
Marianne Grohmann / Agnethe Siquans
}

This article examines texts of the Hebrew Bible about sexual violence as examples of literary transformation. It studies their potential for transformation regarding gender relations in its historical context as well as in contemporary reception. Prescriptive, narrative and poetic texts bring up the issue of sexual violence in different ways and offer starting points for their transformation. The article demonstrates the tight bond between gender and violence in the texts, as well as power and language. It also shows the potential for approaching and interpreting the texts in alternative ways.

Gender; Religion; Transformation; Hebräische Bibel; Sexuelle Gewalt; Tora; Psalmen; Metaphern

Gender; religion; transformation; Hebrew Bible; sexual violence; Torah; Psalms; metaphors

Marianne Grohmann is Professor of Old Testament Studies at the Protestant Faculty of the University of Vienna. Her research activities focus on literary-critical exegesis, Jewish Bible interpretation, gender-fair exegesis, anthropology of the Hebrew Bible; Pentateuch, Psalms and Lamentations. Recent publication: Identität und Schrift. Fortschreibungsprozesse als Mittel religiöser Identitätsbildung (ed. Göttingen 2017).

Agnethe Siquans is Professor of Old Testament Studies at the Faculty of Catholic Theology at the University of Vienna. Her main research areas are inner-biblical exegesis, feminist exegesis, reception of the Old Testament by the Church Fathers, and the relationships between patristic Bible interpretation and Midrash. Currently, she is head of a research project about Exodus 1-2 in patristic and rabbinic interpretation. Recent publication: Die altestamentlichen Prophetinnen in der patristischen Rezeption. TexteKontexte - Hermeneutik (Freiburg 2011).

\section{Sexuelle Gewalt in Gesetzestexten der Tora}

Sexuelle Gewalt wird in der Hebräischen Bibel in Form von Tora, Weisungen oder Gesetzestexten des Pentateuch thematisiert. Beispiele dafür sind Dtn 22,23-29 und eine ältere Parallele dazu aus dem Bundesbuch, Ex 22,15-16. Auch wenn nicht davon auszugehen ist, dass diese Texte eine jemals geltende Rechtslage wiedergeben, sind gesellschaftliche Realitäten in sie eingeflossen: 
Marianne Grohmann / Agnethe Siquans

Das Deuteronomium will in seiner Endfassung eine systematische Gesamtkonstruktion der Gesellschaft Israels sein. Die Gesetze sind demnach - zumindest in der uns vorliegenden Endfassung des Textes - nicht so sehr auf eine konkrete Anwendung ausgerichtet, sondern haben vielmehr einen musterhaften paradigmatischen Charakter. ${ }^{1}$

In Dtn 22,23-29 werden drei Fälle von Vergewaltigung aufgelistet. Die ersten beiden betreffen ein verlobtes Mädchen, der dritte eine junge Frau, die weder verlobt noch verheiratet ist:

\section{Ehebruch und Vergewaltigung eines verlobten Mädchens in der Stadt}

23 Wenn ein Mädchen, eine Jungfrau, einem Mann verlobt ist, und es trifft sie ein Mann in der Stadt und liegt bei ihr (שכב עם škb 'im), 24 dann sollt ihr sie beide zum Tor jener Stadt hinausführen und sie steinigen, dass sie sterben: das Mädchen deshalb, weil es in der Stadt nicht geschrien hat, und den Mann deshalb, weil er der Frau seines Nächsten Gewalt angetan hat. Und du sollst das Böse aus deiner Mitte wegschaffen. ${ }^{2}$

Verlobung gilt im alten Israel als erster Schritt in Richtung Ehe, der Fall wird wie Ehebruch behandelt. „Sowohl Urteil als auch Strafe sind dabei keine ,Erfindung“ der Autoren des Deuteronomiums, sondern die typische Handhabung innerhalb der gesamten altorientalischen Gesetzgebung [...]. “3 Bei der Vergewaltigung eines verlobten Mädchens in der Stadt (Dtn 22,23-24) wird stillschweigendes Einverständnis angenommen und der Fall wie Ehebruch behandelt: Sowohl der Mann als auch das Mädchen werden bestraft.

\section{Ehebruch und Vergewaltigung eines verlobten Mädchens auf dem freien Feld}

25 Wenn aber der Mann das verlobte Mädchen auf dem Feld trifft, und der Mann ergreift sie (והחזיק־בה weh hậ Zîq-bāh) und liegt bei ihr (שכב עם škb 'im), dann soll der Mann, der bei ihr gelegen hat, allein sterben. 26 Aber dem Mädchen sollst du nichts tun, das Mädchen hat keine Sünde begangen zum Tode. Diese Sache ist vielmehr so, wie wenn ein Mann sich erhebt gegen seinen Nächsten und ihn totschlägt. 27 Denn er hat sie auf dem Feld getroffen. Das verlobte Mädchen schrie, aber niemand war da, der es rettete (שעי yš).

Vergewaltigt ein Mann ein verlobtes Mädchen auf dem freien Feld, so wird das als fehlende Zustimmung des Mädchens gedeutet, weil ihr Schrei ungehört geblieben sein kann. In diesem Fall soll nur der Mann bestraft werden (Dtn 22,25-27).

\section{Vergewaltigung eines weder verlobten noch verheirateten Mädchens}

28 Wenn ein Mann ein Mädchen trifft, eine Jungfrau (נערה בתלה na aāāh b tûlāh), die nicht verlobt ist, und ergreift sie (תפש tpś) und liegt bei ihr (שכב עם škb ‘im), und sie werden dabei

1 Paganini 2010, S. 21.

2 Die deutschen Bibeltexte sind, wenn nicht anders angegeben, nach der Elberfelder Bibel zitiert.

3 Paganini 2010, S. 24. 
angetroffen: 29 dann soll der Mann, der bei ihr lag, dem Vater des Mädchens fünfzig Schekel Silber geben, und es soll seine Frau werden, weil er ihr Gewalt angetan hat (ענה 'innāh); er kann sie nicht entlassen all seine Tage.

Die Strafe für die Vergewaltigung einer nicht verlobten Jungfrau ist eine Geldzahlung an den Vater (Dtn 22,28-29), da ein Mädchen vor Verlobung und Ehe dem Vater zugeordnet ist. Zu diesem dritten Fall, der Vergewaltigung eines weder verheirateten noch verlobten Mädchens, gibt es eine ältere Parallele im Bundesbuch, Ex 22,15-16: „15 Wenn jemand eine Jungfrau betört (פתה pth), die nicht verlobt ist, und liegt bei ihr, muss er sie sich gegen das Heiratsgeld zur Frau erwerben. 16 Falls sich ihr Vater hartnäckig weigert, sie ihm zu geben, soll er Geld abwiegen nach dem Heiratsgeld für Jungfrauen."

An beiden Stellen wird ausgedrückt, dass der Vater die Verfügungsgewalt über ein unverheiratetes Mädchen hat. Zwischen Ex 22,15-16 und Dtn 22,28-29 bestehen drei wesentliche Unterschiede:

- Der Kontext ist ein anderer: In Ex 22 werden alle möglichen Fälle von Eigentumsdelikten aufgezählt. Sexueller Kontakt mit einer Jungfrau gilt als Eigentumsdelikt gegenüber dem Vater.

Das Deuteronomium gliedert die Bestimmungen nach dem rechtlichen Status der Frau, die Objekt der sexuellen Handlung ist: die verheiratete Frau, die Verlobte eines anderen Mannes und die unverlobte Frau. Je nach fester Stellung innerhalb der Normalbiographie tun sich mehr oder minder starke Probleme und damit Differenzierungsbedürfnisse der Gesetzgeber auf. ${ }^{4}$

- Die sexuelle Beziehung wird in Ex 22,15 subtiler mit „Verführung / Betören“ (פת pth) beschrieben, in Dtn 22,28-29 explizit als Vergewaltigung bezeichnet.

- Während sich nach dem Bundesbuch der Vater weigern kann, die Tochter zur Ehe herzugeben, ist im Deuteronomium ein Vergewaltiger verpflichtet, das Mädchen zu heiraten. Vielleicht ist in der Haltung des Deuteronomiums ein gewisser Fortschritt, eine „Schutzbestimmung“ zu sehen, weil nicht nur die Rechte des Vaters, sondern auch die des Mädchens auf soziale Absicherung geschützt werden. ${ }^{5}$ In beiden Fällen ist der Vergewaltiger zur Ehe verpflichtet im Bundesbuch kann der Vater dagegen stimmen und seine Tochter ihrem Vergewaltiger nicht preisgeben. Ein gewisser „Fortschritt“ im Deuteronomium liegt vielleicht darin, dass betont wird, dass der Mann die Frau „nicht entlassen kann“. Allerdings ist jede Rede von „Fortschritt“ angesichts des patriarchalen Rahmens dieser Texte äußerst relativ.

\footnotetext{
4 Müllner 1999, S. 49.

5 Vgl. Finsterbusch 2010, S. 378; Paganini 2010, S. 25.
} 
Marianne Grohmann / Agnethe Siquans

Gesetzestexte, rechtliche Bestimmungen der Tora werden also im Lauf der Zeit literarisch transformiert und in andere Kontexte gestellt. Die historische Realität dahinter lässt sich nicht mehr rekonstruieren. Eine Lektüre der Tora-Texte zeigt, dass das Unrechtsbewusstsein für Vergewaltigung im alten Israel ein anderes ist als unser heutiges. In einer Gesellschaft, in der die Sexualität von Mädchen im Besitz ihrer Väter, jene von Frauen ihren Ehemännern zugeordnet ist, gibt es keinen eigenen hebräischen Begriff für „Vergewaltigung“ im heutigen Sinn, d. h. im Sinne einer sexuellen Handlung gegen den Widerstand einer davon betroffenen Person: ,,the physical' use of power by a man in overpowering a woman into non-consensual sexual intercourse." ${ }^{\text {"6 }}$ Aber der Sachverhalt wird immerhin beschrieben: Die Verben, die Vergewaltigung ausdrücken, sind שכב עם škb 'im (schlafen mit), חזק hạ hi. und תפש tāpaś (packen / ergreifen) sowie עם שם innāh pi. (Gewalt ausüben / bedrängen / unterdrücken). ${ }^{7}$ Auch wenn diese Gesetzestexte keine ausdrückliche Definition enthalten, drücken sie doch ein gewisses Verständnis für den Sachverhalt einer Vergewaltigung aus. Die Perspektive von betroffenen Frauen kommt meistens wenig in den Blick. In den Gesetzestexten wird sexuelle Gewalt als Tatbestand konstatiert und in unterschiedlichen literarischen Transformationen dargestellt.

\section{Sexuelle Gewalt in erzählenden Texten}

In den Erzähltexten der Hebräischen Bibel finden sich zwei Kapitel über die Vergewaltigung einer Frau durch einen Mann, Dinas in Gen 34 und Tamars in 2 Sam 13.Dazu kommt Ri 19, wo die namenlose Frau eines Leviten durch eine Gruppe von Männern vergewaltigt wird und schließlich zu Tode kommt. Die Abschnitte der Tora, die sich mit Vergewaltigung befassen (Ex 22,15f.; Dtn 22,23-29) (s. o. 1.), weisen Entsprechungen in der Beschreibung der Geschehnisse zu diesen Erzählungen auf. Im Folgenden werden den Erzählungen über Dina, Tamar und die Frau des Leviten zwei ebenfalls narrative Texte gegenübergestellt, die solche Vergewaltigungsszenen gleichsam umkehren: die Erzählung über Jaël, die den feindlichen Feldherrn Sisera überwältigt (Ri 4-5), und die über eine namenlose Frau, die den Usurpator Abimelech tötet, der ihre Heimatstadt bedroht (Ri 9).

6 Abasili 2011, S. 6.

7 Van Wolde 2002, S. 543, versteht 'innāh allgemeiner als soziale Unterdrückung: ,this verb is used as an evaluative term in a juridical context denoting a spatial movement downwards in a social sense.". 


\subsection{Die Vergewaltigungen Dinas, der Frau des Leviten und Tamars}

Gen 34 erzählt von Dina, die zuerst als Tochter Leas identifiziert wird. Als solche „geht sie hinaus“ (צ׳ yṣ’) und fällt einer Vergewaltigung zum Opfer. Ab diesem Zeitpunkt aber ist sie - aus der Perspektive des Vergewaltigers gesehen - die „Tochter Jakobs“ (V. 3). Eine unverheiratete Frau untersteht der Verfügungsgewalt des Vaters und die Vergewaltigung verletzt daher die Rechte Jakobs. Ebenso werden Verhandlungen über die zu heiratende Frau unter Männern geführt. Nun erzählt Gen 34, dass der Vergewaltiger Sichem nach der Tat an dem Mädchen „hing“. Er liebt sie und spricht zu ihr. Dina spricht nicht. Sichem jedenfalls zieht die (rechtlichen) Konsequenzen aus seiner Tat: Er möchte Dina heiraten und bittet seinen Vater, sich darum zu kümmern. Jakob hört von der Vergewaltigung Dinas. Dann kommt Sichems Vater Hamor, der Landesherr, zu ihm, um über die Heirat zu verhandeln.

Soweit entspricht die Vorgangsweise dem in Dtn 22,28-29 Geforderten:

28 Wenn ein Mann ein Mädchen trifft, eine Jungfrau, die nicht verlobt ist, und ergreift sie und liegt bei ihr, und sie werden dabei angetroffen: 29 dann soll der Mann, der bei ihr lag, dem Vater des Mädchens fünfzig Schekel Silber geben, und es soll seine Frau werden, weil er ihr Gewalt angetan hat; er kann sie nicht entlassen all seine Tage. ${ }^{8}$

Sichem und Hamor halten sich also an die Tora Israels. Sie sind zudem in den Verhandlungen überaus entgegenkommend.

Im Folgenden allerdings treten Dinas Brüder auf, die von den männlichen Untertanen Hamors die Beschneidung verlangen. Sichem und Hamor gehen darauf ein, während der Erzähler die Leser und Leserinnen darüber informiert, dass die Söhne Jakobs hinterhältig handeln. Die Sache endet in einem Blutbad: Sichem, Hamor und ihre männlichen Untertanen werden umgebracht, während sie im Wundfieber liegen. Die Frauen und Kinder aber werden von Dinas Brüdern mitgenommen. Sie begehen damit im Grunde dieselbe Tat, vielfach multipliziert, die sie Sichem und Hamor vorwerfen (Gen 34,31): Sie behandeln die Frauen der Stadt wie „Huren“. Der Erzähler lässt die Leserinnen und Leser nicht im Unklaren darüber, dass er das Vorgehen der Söhne Jakobs nicht billigt.

Was aber passiert mit Dina? Das letzte, was von ihr erzählt wird, ist, dass sie von ihren Brüdern aus dem Haus Sichems geholt wird (Gen 34,26). Das Verb, das dabei verwendet wird (לקח lqh), ist dasselbe, das in V. 2 für das Ergreifen Dinas und in V. 4 für das „Nehmen“ zur Frau gebraucht wird. Dina wird von Männern „genommen“, die sie jeweils in ihre Einflusssphäre bringen wollen. Sie selbst

8 An beiden Stellen wird das Mädchen als נערה (na ${ }^{\text {‘a }}$ rāh) bezeichnet. Für das Ergreifen werden zwei unterschiedliche Verben verwendet (תפש tpś bzw. לקש lqh), Gen 34 gebraucht „liegen mit“ (statt „bei“ wie Dtn 22,28). „Vergewaltigen“ (ענה pi. "innāh) kommt in Dtn 22 nicht in V. 28, aber in V. 24 vor. 
Marianne Grohmann / Agnethe Siquans

kommt mit keiner Silbe zu Wort, sondern ist zum Schweigen verurteilt. Sie wird von ihren Brüdern von ihrem potentiellen Ehemann weggeholt und von da an nur mehr einmal - aber immerhin einmal - erwähnt: in der Genealogie in Gen 46,15, als Jakobs Tochter zusammen mit ihren Brüdern, den Söhnen Leas. ${ }^{9}$

Ri 19-21 ist sicherlich einer der erschreckendsten Texte der Hebräischen Bibel. ${ }^{10}$ Das ist auch durchaus die Absicht dieser Erzählung, die im Kontext des Richterbuches die königslose Zeit als Zeit der Willkür darstellt, in der das Recht des Stärkeren vorherrschte und keine Ordnungsmacht die Gewalt in Schranken weisen konnte. ${ }^{11}$

In Ri 19 geht es um einen Leviten, der ebenso namenlos ist wie seine פילגש (pîlægæš), was in der Regel als „Nebenfrau“ übersetzt wird. ${ }^{12}$ Als die Frau den Leviten verlässt und ins weit entfernte Haus ihres Vaters zurückkehrt, reist er ihr nach. Nach einiger Zeit machen sie sich auf den Rückweg, der Levit, die Frau, ein Knecht und zwei Esel. Als sie vom Einbruch der Nacht überrascht werden, beschließt der Levit, in der israelitischen Stadt Gibea im Gebiet des Stammes Benjamin zu übernachten. Die Bewohner erweisen sich als wenig gastfreundlich, schließlich nimmt ein alter Mann sie in sein Haus auf. Doch dann umringen Männer aus der Stadt das Haus und verlangen die Herausgabe des Gastes - ausdrücklich wird vom „Mann“ gesprochen - um ihn ,zu erkennen“ (V. 22). Mit ידע (yd", „erkennen“) ist Geschlechtsverkehr gemeint. ${ }^{13}$ Der Gastgeber schützt seinen Gast und bietet den Männern seine jungfräuliche Tochter und die Frau des Fremden an. In seinen Worten in V. 23-24 bezeichnet er das Vorhaben der Männer als „Schandtat“ (נבלה n'bālāh; vgl. Dtn 22,21; Gen 34,7; 2 Sam 13,12), von der er sie abhalten will. Allerdings wird in Ri 19 nur die (geplante) Vergewaltigung des männlichen Gastes als solche qualifiziert, nicht die später erfolgende Misshandlung und Vergewaltigung der Nebenfrau. Die jungfräuliche Tochter und die Nebenfrau stehen in der Hierarchie offensichtlich weit unter dem Mann und

9 Die rabbinischen Schriften schreiben Dina eine Heirat mit Simeon (GenR 80,11) oder Ijob (Tanchuma Buber wa-yischlach 19) zu. Eine andere Tradition lässt sie von Sichem schwanger werden und Asenat, die spätere Frau Josefs in Ägypten, gebären (PRE 27).

10 Trible 1990 legt ihn in ihrem Buch, das in der englischen Ausgabe den Titel „Texts of Terror" trägt, ausführlich aus (S. 99-133). Vgl. zur Auslegung auch Scholz 2010, S. 139155.

11 Vgl. dazu Hentschel 2016, S. 277: „Die im Exil formulierte dtr [deuteronomistische] Theologie sieht in den Rettergestalten die bessere Alternative zum gescheiterten Königtum. In nachdtr Zeit lebt die Hoffnung auf eine Fortsetzung der Monarchie wieder auf. Die Zeit, in der es keinen König gab, gilt nun als eine Epoche egoistischer Willkür $(17,6 ; 18,1 ; 19,1 ; 21,25)$.“

12 Die Beziehung eines Mannes zu einer שילגש unterscheidet sich von einem normalen Eheverhältnis. Vgl. Engelken 1989, S. 587.

13 Zu dieser Bedeutung des Verbs und Belegstellen vgl. Botterweck 1982, S. 494. 
erfahren hier keinen Schutz. Im Gegenteil, der Hausherr bietet dem Pöbel explizit die Vergewaltigung der beiden Frauen an. Dabei verwendet er das Verb ענה pi. ('innāh), das auch an anderen Stellen in dieser Bedeutung gebraucht wird (vgl. Dtn 22,24; Gen 34,2; 2 Sam 13,12.14). Nachdem die Männer darauf nicht eingehen, ergreift der Levit seine Frau (ח hzq hi.; vgl. Dtn 22,25.28; 2 Sam 13,11) und stößt sie zu den Männern auf die Straße hinaus. Die Männer „erkennen“ sie, misshandeln sie die ganze Nacht und lassen sie erst am Morgen gehen (V. 25). Sie schleppt sich vor die Haustür des Gastgebers ihres Mannes und fällt dort hin (פל npl; V. 26.27). Der Levit tritt am Morgen vor die Türe, um seine Reise fortzusetzen und sieht dort seine Frau liegen. Die Schilderung dieses Vorgangs ist äußerst befremdlich, da der Eindruck entsteht, dass der Mann gar nicht mehr damit rechnet, seine Frau wiederzusehen. Der Text lässt zudem offen, ob sie zu diesem Zeitpunkt noch lebt oder bereits tot ist. ${ }^{14}$ Jedenfalls reagiert sie nicht auf seine Aufforderung aufzustehen und mit ihm zu gehen. Deshalb legt er sie auf den Esel und bringt sie heim. Die letzten beiden Verse (V.29-30) erzählen den Schluss der Geschichte in aller Kürze, was die Dramatik der Ereignisse umso deutlicher hervortreten lässt. Der Levit nimmt ein Messer, ergreift (wiederum prn hazq hi.) seine Frau und zerschneidet sie in zwölf Teile, die er in das gesamte Gebiet Israels verschickt. Die Reaktion der Augenzeugen spiegelt die erwartete Reaktion der Leser und Leserinnen wider:

Und es geschah, jeder, der es sah, sagte: So etwas wie das hier ist nicht geschehen, noch wurde es gesehen seit dem Tag, da die Söhne Israel aus dem Land Ägypten heraufgezogen sind, bis zu diesem Tag. Richtet euer Herz darauf, beratet und redet! (Ri 19,30)

Mit den Imperativen am Ende wird ein Urteil der Rezipierenden - derer, die die Teile der Frauenleiche erhalten, aber auch derer, die diese Erzählung lesen herausgefordert.

Die nächsten beiden Kapitel des Richterbuches schildern die Reaktion der Israeliten, die in einem Blutbad israelitischer Stämme gegeneinander und einem Frauenraub resultiert. ${ }^{15}$ Von Bedeutung ist aber nicht in erster Linie die vergewaltigte und getötete Frau, sondern die Beziehungen der Männer der unterschiedlichen Gruppen zueinander: „Mit der Ausrottung von Benjamin wird nicht der elende Tod einer namenlosen Frau gerächt, sondern die dadurch verletzte ,Ehre' eines Mannes, der sich schlechterdings ehrlos benimmt. Um den fast ausgerotteten Stamm Benjamin wiederherzustellen, werden am Ende 400 Frauen zwangsverheiratet und 200 weitere gekidnappt, wird also die Banden-Verge-

14 Vgl. dazu Trible 1990, S. 117 f.

15 Die Jungfrauen werden bei einem Fest geraubt, das auf Ri 11,37-40 zurückgeht. Sie gedenken alljährlich der Tochter Jiftachs, die von ihrem Vater aufgrund eines Gelübdes als Opfer dargebracht worden war. 
Marianne Grohmann / Agnethe Siquans

waltigung einer Frau in 19 durch die Massenvergewaltigung von 600 Frauen in 21 ,kompensiert'.“16

Im Zuge der Beratungen stellt der Levit die Ereignisse in Gibea aus seiner Perspektive dar: „Mich wollten sie töten und meine Nebenfrau vergewaltigten sie, sodass sie starb.“ (Ri 20,5) Dass der Levit hier von einer Tötungsabsicht ihm gegenüber spricht, verschleiert, vielleicht bewusst, die Vergewaltigungsabsicht der Männer von Gibea. Eine Vergewaltigung ist immer eine Demütigung, die angesichts des hierarchischen Geschlechterverhältnisses für einen Mann eine noch drastischere Erniedrigung mit sich bringt, als bei einer Frau. Dass die Vergewaltigung der Frau dann tatsächlich mit dem Tod endet, bestätigt die Befürchtung des Mannes, die sich in seiner Erzählung retrospektiv ausdrückt. Der Tod der Frau wird eindeutig mit der Vergewaltigung (ענה pi. 'innāh) in Zusammenhang gebracht, die hier erstmals vom Ehemann als solche angesprochen wird. In V. 6 bezeichnet er das Geschehene als „Schandtat“, was in der Antwort der versammelten Israeliten noch einmal bestätigt wird (V. 10).

Vieles in dieser Erzählung bleibt offen oder zweideutig. Letztlich zielt der Text auf die unhaltbaren Zustände in Israel, die Schandtat und Gewalt zwischen den Stämmen hervorrufen. Die Frau, die am Anfang als aktives Subjekt auftritt, wird zum Objekt, sobald der Mann das Haus ihres Vaters betritt. Die einzigen ihr später noch zugeschriebenen Handlungen finden sich in Ri 19,26, wo sie am Morgen zum Haus, in dem ihr Mann Gast ist, kommt und hinfällt. Wie Dina ist auch diese Frau sprachlos und noch dazu namenlos. Sie verschwindet mit ihrer Zerstückelung aus der (biblischen) Geschichte, allein unmittelbar danach, in Ri 20, wird das Geschehen noch einmal aufgerollt und explizit als Vergewaltigung und Schandtat qualifiziert. ${ }^{17}$ Paradoxerweise wird die Untat gerade durch den Tod der Frau und das Verschicken ihrer Leichenteile im ganzen Land bekannt und verurteilt, während Dina und Tamar im Schweigen versinken. Die Reaktion aber ist hier wie nach den Vergewaltigungen Dinas und Tamars weitere Gewalt unter Männern, ja sogar unter brüderlichen Gruppen innerhalb Israels.

Die Ermordung der Täter, aber auch der Tod der Frau sind angesichts der Tora allerdings logische Folgen. Im Unterschied zu Dina und Tamar ist die Frau des Leviten keine unverheiratete Jungfrau. Die in Dtn 22,23-29 erörterten Tatbestände treffen in diesem Fall nicht zu. Im Grunde begehen die Männer von Gibea Ehebruch, der nach Dtn 22,22 die Todesstrafe nach sich zieht. Demnach wird auch die beteiligte Frau mit dem Tod bestraft. Andererseits wird - zumindest in Ri 20,5 - eindeutig von Vergewaltigung gesprochen, niemals von Ehebruch. Im Falle einer verlobten Frau (für eine Ehefrau oder Nebenfrau gibt es diese Regelung

16 Knauf 2016, S. 162.

17 Hos 9,9 und 10,9 erwähnen die „Tage von Gibea“ im Zusammenhang der Sünde Israels. Damit bezieht sich Hos im Lesegefälle der Hebräischen Bibel wohl auf Ri 19. Das ist allerdings nicht mit der entstehungsgeschichtlichen Reihenfolge der Texte gleichzusetzen. Vgl. zum Verhältnis dieser beiden Texte Groß 2009, S. 878-879. 
nicht), die in der Stadt mit einem anderen Mann Geschlechtsverkehr hat, sollen wiederum beide sterben (vgl. Dtn 22,23-24). In Ri 19 ist jedoch klar, dass die Frau keineswegs zugestimmt hat, selbst wenn sie nicht um Hilfe geschrien hätte, sondern ihr eigener Mann hat sie „ergriffen“ - das Verb wird sonst mit dem Vergewaltiger als Subjekt verwendet - und sie den Gewalttätern übergeben. Damit ist nicht so eindeutig, wen tatsächlich die Schuld trifft. Der Gastgeber und der Levit sind jedenfalls an der Vergewaltigung und dem Tod der Frau beteiligt, kommen aber ungeschoren davon. Der Erzähler des Textes lässt jedoch deutlich erkennen, dass er bei allen Beteiligten Unrecht sieht. Die Tora jedenfalls ist hier nicht Maßstab des Handelns. Ri 21,25 schließt die Perikope mit den Worten ab: „In jenen Tagen war kein König in Israel. Jeder tat, was recht war in seinen Augen." Es handelt sich um eine rechtlose Zeit, was auch und besonders die Frauen am eigenen Leib erfahren müssen.

Von der Vergewaltigung Tamars, einer Tochter Davids, durch ihren Bruder oder Halbbruder Amnon erzählt 2 Sam 13. ${ }^{18}$

Amnon begehrt Tamar, hat aber immerhin Bedenken, ihr etwas anzutun, da sie noch Jungfrau ist, und das heißt, dass sie der Verfügungsgewalt des gemeinsamen Vaters David untersteht. Amnons Freund und Cousin Jonadab stachelt Amnon aber an, mit Hilfe einer vorgetäuschten Krankheit Tamar in seine Nähe zu locken und so zu seinem Ziel zu kommen. Als Tamar ihm Kuchen gebacken hat, schickt er alle aus dem Zimmer und lockt Tamar noch näher zu sich heran. Dann heißt es (V.11): ,[...] er packte sie (ויחזק־ב wayyah ${ }^{\mathrm{a} z æ q}$ bāh) und sagte zu ihr: Komm, liege bei mir, meine Schwester!" (שכב עם Škb 'im, wie in Dtn 22,28). Tamar aber versucht das Schlimmste abzuwenden. „Nicht doch, mein Bruder! Tu mir keine Gewalt an (ענה pi. 'innāh), denn so handelt man nicht in Israel! Tu doch diese Schandtat nicht!“(V.12) Sie verweist auf ihrer beider Rufschädigung, die eine solche Tat zur Folge haben würde, und schlägt ihm vor, den König, also ihrer beider Vater, um eine Heiratserlaubnis zu bitten. Aber Amnon hört nicht auf sie, packt sie, vergewaltigt sie und liegt bei ihr. Wiederum werden die drei Verben חעי (hzq), עזק pi. ('innāh) und שכב את (škb 'et) verwendet. Wie diese Tat einzuordnen ist, ist damit klar: Es handelt sich um eine Vergewaltigung. Die Fortsetzung der Geschichte aber weicht zunächst drastisch von der Dinas ab: Nach der Tat wird Amnon von Hass auf Tamar erfasst und wirft sie hinaus. Sie aber macht ihre Vergewaltigung durch öffentliches Schreien, Zerreißen ihres Gewandes und weitere Trauergesten offenbar (V. 19). Ihr Bruder Abschalom versteht auch sogleich, was passiert ist. Erschütternd ist allerdings seine Reaktion: Er fordert Tamar zum Schweigen auf und schickt sie in sein Haus. Später nimmt er die Rache für seine Schwester in die eigene Hand, da der Vater David keine Schritte setzt. ${ }^{19}$ Damit wird wiederum eine

18 Wer Tamars Mutter ist, erwähnt die Hebräische Bibel an keiner Stelle. Daher ist ihr genaues Verhältnis zu Amnon, ebenso wie zu ihrem anderen Bruder Abschalom, unklar. Vgl. zu dieser Erzählung ausführlich Müllner 1997.

19 Fokkelman deutet Abschaloms Verhalten positiv. Fokkelman 1981, S. 110: „Ab- 
Marianne Grohmann / Agnethe Siquans

Spirale der Gewalt in Gang gesetzt, die im Grunde bereits ihren Anfang in Davids Ehebruch mit Batseba, der Frau des Urija (vgl. 2 Sam 11) - möglicherweise ist auch diese Episode als Vergewaltigung zu deuten ${ }^{20}-$ genommen hat und die gesamte Thronfolgegeschichte Davids bis 1 Kön 2 durchzieht.

Was erfahren die Leserinnen und Leser über das weitere Schicksal Tamars? 2 Sam 13,20 informiert darüber, dass Tamar von diesem Zeitpunkt an im Haus ihres Bruders Abschalom שממה (šom ${ }^{\mathrm{e}} \mathrm{māh}$ ) lebte. ${ }^{21}$ Meist wird dieses Adjektiv als „einsam“ oder „verlassen“ übersetzt. An anderen Stellen aber bedeutet es „verwüstet", „öde“. Diese Beschreibung wäre dann auf Tamars Zustand nach der Vergewaltigung zu beziehen. Noch einmal wird auch der Name Tamars erwähnt, nämlich im folgenden Kapitel (2 Sam 14,27). Dort werden Abschaloms Kinder aufgezählt: Er hatte drei Söhne, deren Namen überraschender Weise den Leserinnen und Lesern vorenthalten werden, und eine Tochter namens Tamar, die sehr schön wurde, wie es heißt. U. E. ist es nicht ausgeschlossen, dass die Mutter dieser Kinder die vergewaltigte Tamar ist, die fortan in Abschaloms Haus als seine Frau lebt. Das wird keineswegs explizit gesagt, aber die Erzählung enthält hier Leerstellen, die eine solche Deutung zumindest nicht ausschließen. 2 Sam schweigt darüber, was weiterhin mit Tamar geschieht, zudem wird kein Name einer anderen Frau Abschaloms bzw. der Mutter seiner Kinder genannt.

Diese drei Erzählungen, die sich explizit mit der Vergewaltigung von Frauen beschäftigen und diese Erfahrung benennen - auch wenn die vergewaltigten Frauen selbst nicht zu Wort kommen -, haben noch eines gemeinsam: Der Gewalt gegen die junge Frau folgen Gewaltexzesse unter den Männern. ${ }^{22}$

Auffällig ist außerdem, dass in diesen Erzählungen, die ja immerhin als Teil der Geschichte des Volkes JHWHs, Israel, überliefert wurden, dieser Gott keine Rolle spielt. Er scheint den Ereignissen fern zu sein.

salom receives his sister. Undoubtedly, he will have tried to console her as best he can as is evinced by the vague and, therefore, soothing terms with which he describes rather than mentions the rape [...] But consolation is not the foremost meaning of this discourse in v. 20. [...] In other words, Absalom first puts a stop to Tamar's loud clamour and then lets her know that by means of this contrast he wants to avoid her doing something about her rape herself. [...] Absalom wants to take the law into his own hands." Müllner 1997, S. 315-321, diskutiert die unterschiedlichen Deutungen von 2 Sam 13,20 und zieht den Schluss: „Der verbale Gewaltakt Abschaloms erreicht, was der sexuelle Gewaltakt Amnons nicht erreichen konnte: den Tod dieser Frau.“ (S. 321).

20 Vgl. etwa Abasili 2011.

21 Vgl. zur Bedeutung dieses Adjektivs in 2 Sam 13,20 Müllner 1997, S. 323-327. Müllner arbeitet zwei wesentliche Charakteristika heraus, die der Begriff transportiert: Schmach und Ortlosigkeit. Diese Ortlosigkeit stehe in eigenartigem Kontrast zu ihrem Aufenthaltsort im „Haus“, das normalerweise Sicherheit und Versorgung repräsentiert (vgl. etwa Rut 1,8.9).

22 Vgl. dazu Yamada 2008. 


\section{2. „Reversed Rape“: Die „Vergewaltigung“ Siseras und Abimelechs}

In der Hebräischen Bibel finden sich auch zwei Erzählungen, die das Vergewaltigungsmuster aufgreifen und transformieren. Ri 4-5 präsentiert die Unterdrückung Israels durch die Kanaanäer und die Errettung aus dieser Notsituation durch Debora, Barak und Jaël: Letztere tötet den kanaanäischen Feldherrn Sisera. Ri 4 ist eine narrative Darstellung, während Ri 5 die Ereignisse hymnisch resümiert und interpretiert. ${ }^{23}$

In Ri 4,9 prophezeit die Richterin und Prophetin Debora dem israelitischen Feldherrn Barak: „JHWH wird Sisera der Hand einer Frau ausliefern.“ Prophetien sind immer uneindeutig. So ist auch hier nicht klar, wer diese Frau sein wird: Debora oder eine andere? Die Erzählung geht dabei ganz von einer androzentrischen Kriegsideologie aus, in der die siegreichen Helden gepriesen werden und die Vergewaltigung von Frauen im Gefolge eines Sieges als der Normalfall erscheint. ${ }^{24}$ Dass ein Mann, der von einer Frau besiegt wird, in besonderer Weise gedemütigt wird, passt in diese Ideologie. In einem Konzept von Ehre vs. Schande ist der Tod in der Schlacht ehrenvoll, die Ermordung durch eine Frau, noch dazu im Schlaf, liegend, dagegen schändlich. ${ }^{25}$ Diese Schande trifft selbstverständlich nicht nur den getöteten Sisera, sondern auch Barak, dessen Ruhm durch die Tat der Frau zerstört wird.

Dennoch wird das Muster sexueller Gewalt von Männern gegen Frauen in dieser Erzählung transformiert und kann als „,reversed rape“ verstanden werden. ${ }^{26}$ Mütterliche und sexuelle Metaphorik werden nebeneinander gebraucht. Jaël nimmt Sisera schützend auf und nährt ihn, wobei diesen Tätigkeiten mütterliche Konnotationen zugeschrieben werden. Das Zelt Jaëls, in das Sisera eintritt, kann aber auch als ihr Körper gedeutet werden. Wasser wird immer wieder als Symbol für männliche oder weibliche Sexualität verwendet (vgl. Spr 5,15.16; 9,17; Hld 4,15). Das Verb „̈̈ffnen“ (פ פth) wird auch für das Öffnen des Mutterschoßes und damit für weibliche Fruchtbarkeit gebraucht (vgl. Gen 29,31; 30,22), aber ebenso für das Öffnen der Tür für den Geliebten (vgl. Hld 5,2.5.6). Die

23 Mit Fewell / Gunn 1990 lese ich die beiden Texte in der in der Hebräischen Bibel gegebenen Reihenfolge. Zu den Unterschieden zwischen beiden Darstellungen vgl. ebd., S. 399-402.

24 Vgl. etwa Exum 1999, S. 92-95. Exum sieht den Text Ri 4-5 als ganzen in der männlichen Ideologie befangen. Auch den Frauen wird diese in den Mund gelegt (und damit nahegelegt). Exum deutet Debora und Jaël als die gute und die tötende Mutter. Dieser Aspekt ist bemerkenswert, hier soll allerdings der Fokus auf die Frage der Vergewaltigung gelegt werden.

25 Vgl. dazu Bal 1988, S. 116-124. Bal ist der Ansicht, dass der implied author von Ri 4 maskulin ist, von Ri 5 feminin (S. 115).

26 Vgl. Fewell / Gunn 1990, S. 394. Vgl. auch Bal 1988, S. 131-134. Nach Bal überschneiden sich in Ri 5 mehrere Bedeutungslinien, zu denen auch die sexuelle gehört. 
Marianne Grohmann / Agnethe Siquans

Wendung „sie kam zu ihm“ (תבוא אליו tābō' 'elāyw; vgl. aber 2 Sam 11,4) kehrt das übliche „,er kam zu ihr“ um, das sich ebenfalls im Kontext von Geschlechtsverkehr findet (vgl. z. B. Ez 23,44; Gen 38,16; Ri 15,1; 2 Sam 11,11; 16,21). Fewell und Gunn kommentieren: „Sisera, like a man penetrating his lover, has entered, upon invitation, a woman's sphere. Now, like a child in a womb, Sisera lies sleeping in Jael's tent.“27 Sisera hat die Grenze zur ,weiblichen“ Sphäre überschritten; umgekehrt überschreitet Jaël die Grenze in Richtung der „männlichen“ Sphäre, ${ }^{28}$ die durch Gewalt im Krieg gekennzeichnet ist.

Jaëls Mord erfüllt die Prophetie Deboras und beschämt sowohl Barak als auch Sisera. Ihr entscheidendes Charakteristikum, das sie für diese Tat prädestiniert, ist, dass sie eine Frau ist. Als Frau und nur als Frau kann sie dieses Ende der Geschichte herbeiführen. Dass ihr der Mord gelingt, ist nur der patriarchalen Ideologie zu verdanken, die in einer Frau ausschließlich die Mutter oder die potentielle Sexualpartnerin sieht. Ri 4 macht klar, dass es so nicht immer ist.

Interessanter Weise wird die Tat Jaëls mit dem Wirken Gottes in Verbindung gebracht. „So demütigte Gott an diesem Tag Jabin, den König von Kanaan, vor den Israeliten." (Ri 4,23) Jaël geht es, obwohl sie vermutlich keine Israelitin ist, um das Wohl, das Überleben des Volkes Israel, nicht allein um ihr eigenes Wohl. Selbst wenn man die Episode so deuten wollte, dass sie sich gegen ihre Vergewaltigung durch Sisera wehrte, ist das Ergebnis die Befreiung ganz Israels von der Bedrohung durch die Kanaanäer.

Der Hymnus in Ri 5 resümiert die erzählten Ereignisse in poetischer Weise mit eigenen Akzenten. Auch in dieser Version nimmt die Tat Jaëls einen wichtigen Platz ein. ${ }^{29}$ Sie ist Subjekt der Handlungen, Sisera das Objekt. Auch diese Darstellung ist als umgekehrte Vergewaltigung zu verstehen. Den Anhaltspunkt dafür bietet die Schilderung von Siseras Tod in Ri 5,24-27:

Gesegnet vor allen Frauen sei Jael, die Frau Hebers, des Keniters, vor allen Frauen im Zelt sei sie gesegnet! Wasser verlangte er, Milch gab sie; in einer kostbaren Schale reichte sie Rahm. Ihre Hand streckte sie aus nach dem Pflock, und ihre Rechte nach dem Arbeitshammer. Und sie schlug Sisera mit dem Hammer, zerschmetterte sein Haupt, sie zerschlug und durchbohrte seine Schläfe. Zwischen ihren Füßen krümmte er sich, fiel, lag da; zwischen ihren Füßen krümmte er sich, fiel; da, wo er sich krümmte, fiel er - vernichtet.

Das dreimalige „Fallen“ in V. 27 beschreibt Siseras Demütigung bis hin zu seiner Vernichtung. ${ }^{30}$ Das Eindringen der Frau mit dem Zeltpflock in den Körper des

27 Fewell / Gunn 1990, S. 393.

28 Was jeweils als „,weibliche“ und „männliche“ Sphäre angesehen wird, ist eine Frage der kulturellen Interpretation und Definition.

29 Vgl. dazu Bal 1988, S. 127-134.

30 Zuvor ist Sisera bereits vom Streitwagen herabgestiegen (V. 15). 
Mannes, sowohl in Ri 4,21 als auch in 5,26, weist sexuelle Konnotationen auf, wobei die üblichen Rollen hier umgekehrt werden. Die Füße werden in der Hebräischen Bibel auch als Euphemismus für die Geschlechtsorgane verwendet, ${ }^{31}$ sodass hier ebenfalls eine sexuelle Konnotation angenommen werden kann. Sisera liegt zwar bei Jaëls Geschlechtsorganen, ist aber „gefallen“ (dreimaliges npl). „Between the women's legs a man lies dead - a rather sobering image to any man, no matter which side of the war he fights on.“32 In diesem Bild wird tatsächlich die androzentrische Struktur durchbrochen, ja geradezu umgekehrt, zumindest für einen Augenblick.

Ein weiterer Hinweis auf die Verbindung der Szene mit einer Vergewaltigung ist die Erwartung von Siseras Mutter und den Frauen um sie: Ri 5,30 berichtet davon, dass die Mutter Siseras und die Fürstinnen auf seine Rückkehr warten. Sie sprechen die Vermutung aus, dass er sich deswegen verspätet habe, weil die Krieger noch auf Beutezug sind. Die Beute besteht unter anderem aus ein oder zwei Frauen (wörtlich: רחם ræhæm, „Mutterschoß“) für jeden Mann. Das, was seine Mutter erwartet, ist gerade nicht eingetreten, sondern hat sich durch die Tat Jaëls umgekehrt. Ergebnis dieser umgekehrten Vergewaltigung (oder vielleicht auch der Verhinderung von Vergewaltigung) sind 40 Jahre der Ruhe für das Volk Israel, und das meint hier: keine Gewalt und keine Kriegshandlungen, aber für Frauen natürlich auch keine (drohende) Vergewaltigung. Der patriarchale Kontext wird punktuell unterbrochen, um ihn auf Dauer aufrecht zu erhalten.

Auch Abimelech, der von einer Frau durch einen Mühlstein getötet wird, wird Opfer sexuell konnotierter Gewalt, die wiederum die üblichen Machtverhältnisse und Rollen umkehrt (Ri 9,50-54; vgl. 2 Sam 11,21). ${ }^{33}$ Ri 9,50-54 beschreibt die Tötung Abimelechs durch eine namenlose Frau:

50 Dann zog Abimelech nach Tebez und belagerte Tebez und nahm es ein. 51 Es war aber ein starker Turm mitten in der Stadt, und dahin flohen alle Männer und Frauen, alle Bürger der Stadt. Und sie schlossen hinter sich zu und stiegen auf das Dach des Turmes. 52 Und Abimelech kam an den Turm und kämpfte gegen ihn. Und er trat an den Eingang des Turmes, um ihn mit Feuer zu verbrennen. 53 Da warf eine Frau einen oberen Mühlstein auf Abimelechs Kopf und zerschmetterte ihm den Schädel. 54 Da rief er schnell nach dem Burschen, seinem Waffenträger, und sagte zu ihm: Zieh dein Schwert und töte mich, damit man nicht von mir sagt: Eine Frau hat ihn erschlagen! Und sein Bursche durchbohrte ihn, daß er starb.

Die Frau verwendet einen Mühlstein, und zwar einen oberen Mühlstein (פלח רכב pælạ rækæb), um Abimelech zu töten. Die Arbeit mit der Mühle war eine alltägliche Tätigkeit von Frauen. Athalya Brenner hat aber auch auf die sexuelle

31 Vgl. Ex 4,25; Jer 7,20; Ez 16,25; Rut 3,7; ev. Jes 6,2.

32 Fewell / Gunn 1990, S. 405.

33 Vgl. zum Folgenden Brenner 1993, S. 143-145. 
Marianne Grohmann / Agnethe Siquans

Symbolik der Mühlsteine hingewiesen. Das Mahlen beider Steine aneinander stellt bildlich den sexuellen Akt dar. Dabei ist - man möchte sagen, selbstverständlich - der obere Mühlstein dem Mann zugeordnet. Wenn nun die Frau aus der Stadt Tebez einen oberen Mühlstein verwendet, dann nimmt sie die Position des Mannes ein, die übergeordnete, aktive Position. Daraus folgt, dass Abimelech, der unterhalb des Turmes steht und auf den der Mühlstein von oben herab fällt, nun in die untergeordnete, passive Position, die einer Frau, gezwungen wird. Er erkennt dies sofort: Von einer Frau auf diese Weise getötet zu werden, ist erniedrigend. So bittet er seinen Waffenträger, ihn auf männliche Weise zu töten. Dennoch erinnert 2 Sam 11,21 gerade an dieses Detail: Abimelech wurde von einer Frau mit einem oberen Mühlstein getötet. Damit bietet die Hebräische Bibel zwei Deutungen des Todes Abimelechs, die aber beide die Schande von einer Frau getötet zu werden thematisieren.

Auch im Anschluss an diese Episode tritt zunächst Ruhe ein: Die Israeliten können nach Hause zurückkehren, der Krieg ist vorüber. V. 56 schließt die Erzählung mit den Worten ab: „So ließ Gott die Bosheit Abimelechs, die er an seinem Vater verübt hatte, indem er seine siebzig Brüder erschlug, auf ihn zurückkommen." Der Gewalttäter ist in Schande und nicht ehrenvoll zugrunde gegangen. Wie in Ri 4-5 ist die Konsequenz dieser umgekehrten Vergewaltigung ein Ende der Gewalt (anstelle von Eskalation von Gewalt wie bei Dina, der Frau des Leviten und Tamar). In beiden Erzählungen wird die Tat der Frau mit Gott verknüpft, der in den anderen Vergewaltigungserzählungen auffällig abwesend ist.

\subsection{Transformation der Geschlechterverhältnisse?}

Sexuelle Gewalt, in wörtlichem und in metaphorischem Verständnis, spiegelt und symbolisiert Machtverhältnisse. Bei den Vergewaltigungen Dinas, der Frau des Leviten und Tamars geht es immer um die Machtkonstellationen zwischen Männern bzw. von Männern repräsentierten Gruppen. Die vergewaltigten Frauen erscheinen als wertvolles Gut in diesen Konflikten, aber als Objekte, denen kaum Handlungsmacht oder eine Stimme zugestanden wird. Vor allem nach der Vergewaltigung schweigen und verschwinden die Frauen. Im Rückblick erscheinen sie gerade noch als Anlass für Gewaltexzesse ihrer Väter, Ehemänner und Brüder. In 2 Sam 13,12 stellt ausgerechnet Tamar fest: „So etwas tut man nicht in Israel.“ Sie qualifiziert die Vergewaltigung als „Schandtat“ (נבלה n bālāh; vgl. Dtn 22,21; Gen 34,7; Ri 19,23.24). Die Vergewaltigung einer Jungfrau verletzt die Rechte ihres Vaters; jede Vergewaltigung einer Frau verletzt Rechte eines anderen Mannes. Und damit steht die Stabilität der sozialen Ordnung auf dem Spiel. Gerade vor dem Hintergrund einer geschwisterlichen/brüderlichen Gesellschaft, wie sie das Deuteronomium entwirft, ist so etwas undenkbar. In Gen 34,7 wird Sichem von Dinas Brüdern bezichtigt, eine Schandtat begangen zu haben. Die 
Folge daraus ist, dass solch eine Sippe sich nicht mit der Sippe Jakobs verschwägern und verbrüdern darf. ${ }^{34}$ Die vergewaltigten Frauen repräsentieren die Gemeinschaft, der sie angehören. ${ }^{35}$ Wenn ein Mann die Schandtat einer Vergewaltigung begeht, resultieren daraus Gewalt, Spaltung und letztlich die Zerstörung der Gemeinschaft. Das wird als nicht gottgewollt qualifiziert, wie die Abwesenheit Gottes in diesen Erzählungen deutlich macht.

Die Episoden in Ri 4-5 und Ri 9 kehren die üblichen Machtverhältnisse zwischen Frauen und Männern um und transformieren sie dadurch zumindest punktuell. In beiden Erzählungen wird diese Umkehrung auch thematisiert: Von einer Frau getötet zu werden, ist besonders demütigend für einen Mann, besonders für einen Krieger. In der Regel, so Ri 5, sind es die Krieger, die sich der besiegten Frauen bemächtigen. Die Texte verbleiben im Rahmen androzentrischer Ideologie und letztlich wird die patriarchale Ordnung wiederhergestellt. Sie funktionieren auch nur in diesem Rahmen, denn nur unter der Annahme, dass Frauen mütterlich oder als passive Geliebte handeln (und nicht töten), also in der ihnen zugedachten Position bleiben, können sie ausnahmsweise diese Grenzen überschreiten und damit die männlichen Kontrahenten an einem Schwachpunkt überraschen.

Warum werden solche Erzählungen aber überliefert? Beide Frauen handeln im Dienste der Gemeinschaft, in der sie leben. Das Ergebnis ihres Handelns ist die Beendigung eines Krieges und Frieden. In beiden Erzählungen wird der Sieg der Frauen auf Gott zurückgeführt. Das kann einerseits so verstanden werden, dass beide Frauen diese Siege gar nicht aus eigener Kraft erringen könnten oder konnten. ${ }^{36}$ Andererseits - und das ist wohl dem Kontext angemessener - werden sie als diejenigen gesehen, die in Einklang mit Gott und in seinem Sinne handeln. Die Frauen repräsentieren eine (militärisch) bedrohte, unterlegene, aber dennoch in ihren Vorstellungen patriarchal geprägte Gemeinschaft. So wird ja besonders in prophetischen Texten das Volk Israel oder die Stadt Jerusalem durch eine Frau (mitunter auch eine Frau, die sexuelle Gewalt erfahren muss; vgl. Ez 16;23 u. a. ${ }^{37}$ ) verkörpert. Hier handelt es sich freilich um individuelle Gestalten, die aber dennoch nicht für sich allein stehen. Sie handeln für die Gemeinschaft und sie repräsentieren zugleich als Frauen die Schwäche und Unterlegenheit dieser Gemeinschaft. Dabei überwinden sie aber die ,weibliche“ Sphäre und dringen in den „männlichen“ Bereich vor. Sie töten die feindlichen Krieger explizit als Frauen und nur, weil sie Frauen sind. Das zeigt sich etwa auch darin, dass die Frau, die

34 Gen 34,29 spricht davon, dass die Söhne Jakobs auch die hiwitischen Frauen geraubt hätten. Diese der Vergewaltigung gleichwertige Tat wird im Text nicht problematisiert.

35 Zur Vergewaltigung von Frauen als Metapher für die Zerstörung einer Gemeinschaft vgl. Keefe 1993.

36 Das trifft aber auch auf Männer zu, wie etwa Ri 7 sehr anschaulich verdeutlicht.

37 Vgl. zur sexuellen Metaphorik in Ez Jost / Seifert 1999, S. 286-289. 
Marianne Grohmann / Agnethe Siquans

Abimelech tötet, keinen Namen hat. Sie benötigt ihn nicht, da ihr entscheidendes Charakteristikum ihr Frau-Sein ist. Letztlich geht es nicht um diese Frauen, sondern um die Erniedrigung der Feinde Gottes. Es geht um den vom Gott Israels unterstützten Sieg „,des Schwachen“. Die damit transportierte Botschaft liegt im Interesse der Tradentengruppe, die ihre Identität in diesen Texten definiert und daraus wiederum als hoffnungsvolle Zukunftsperspektive bestärkt.

Diese Durchbrechungen der sozialen Ordnung sind in der Hebräischen Bibel punktuell anzutreffen, lassen eine Alternative aufscheinen - wohlgemerkt nur in Ausnahmefällen als letzten Ausweg! -, aber transformieren diese nicht nachhaltig. Im Gegenteil: Zielperspektive aller dieser Texte ist die Wiederherstellung der verlorenen Machtposition, die durch die Männer repräsentiert wird. Es findet in diesen Texten keine grundsätzliche und nachhaltige Transformation von Geschlechterverhältnissen oder auch von Gewalt statt.

Aber - und das ist unbedingt festzuhalten - sowohl die sexuelle Gewalt eines Mannes an einer Frau als auch deren Transformation werden erzählerisch festgehalten. Das Leiden und die Taten aller dieser Frauen, derer, die Gewalt erleiden, und derer, die Gewalt ausüben und somit eingefahrene Verhältnisse umkehren, ${ }^{38}$ werden erinnert, narrativ wiederholt und damit aktualisierbar. Zu allen vorgestellten Texten gibt es je einen „Erinnerungstext“: Im Falle der Erzählung von Ri 4 ist es der Hymnus in Ri 5, der Jaëls ruhmvolle Tat besingt. Die Frau, die Abimelech tötet, wird später im Zusammenhang mit einer Erinnerung an Abimelechs Tod noch einmal erwähnt (2 Sam 11,21). Dina findet in einer Genealogie Erwähnung (Gen 46,15), wo sonst in der Regel nur die Söhne aufgezählt werden. Sogar Tamar wird erinnert, im Namen ihrer Nichte (2 Sam 14,27). Die Vergewaltigung der Frau des Leviten wird durch diesen selbst ganz Israel bekannt gemacht (Ri 20) und Hosea nimmt Bezug auf die „Tage von Gibea“ (Hos 9,9; 10,9). ${ }^{39}$ Damit werden auch die schamhaft verschwiegenen vergewaltigten Frauen zumindest ein klein wenig dem Vergessen entrissen, und Gegenstimmen, vielleicht sogar Stimmen von Frauen, werden hörbar. Religiöse Texte, besonders „kanonische“ Texte wirken prinzipiell bewahrend. Die Hebräische Bibel ist aber dadurch charakterisiert, dass sie auch solche Gegenstimmen bewahrt, wie subtil auch immer. Diese Gegenstimmen eröffnen den Rezipientinnen und Rezipienten die Möglichkeit, die Verhältnisse auch anders zu denken, und bieten damit einen Anhaltspunkt für ein Hinterfragen und eine Transformation der Geschlechterund Machtverhältnisse.

38 Dabei spielt es keine Rolle, ob die erzählten Ereignisse und die involvierten Personen historisch sind oder nicht.

39 Zwar werden die Frau und ihre Vergewaltigung hier nicht eigens erwähnt, sie sind aber unverzichtbarer Teil der Geschehnisse, auf die Hos anspielt. Wenngleich Hos 9,9 und 10,9 möglicherweise dem Autor von Ri 19 schon vorgelegen ist (vgl. Groß 2009, S. 878-879), ist im vorliegenden Bibeltext Hos als retrospektive Anspielung auf die Ereignisse von Ri 19-21 zu lesen. 


\section{Poetische Transformationen von Gewalterfahrungen}

Die Psalmen und Klagelieder der Hebräischen Bibel sind genauso wie rechtliche Bestimmungen und Erzählungen in langen literarischen Transformationsprozessen entstanden. Während gesetzliche und narrative Texte sexuelle Gewalt darstellen oder bewerten, transformieren Psalmen Gewalt in poetische Sprache. Psalmen stellen eine Sprache zur Verfügung, um vielfältige Erfahrungen, darunter auch Gewalt, zur Sprache zu bringen. In Gebetssprache wird Gott direkt angesprochen. Es liegt in der großen Deutungsoffenheit poetischer Texte begründet, dass sie zu vielfältigen Transformationsprozessen auf der Ebene der Interpretation anregen. Als zwei Beispiele poetischer Texte, in denen Gewalterfahrungen thematisiert werden, sollen hier Ausschnitte aus Psalm 55 und Klagelied 1 gelesen werden.

\subsection{Psalm 55}

Das „Klagelied“40 oder „Gebetslied“"41 Ps 55, „die Vertextung eines gegliederten Klageprozesses “42 ist wie alle Psalmen mehrdeutig in verschiedene Richtungen lesbar. An vielen Stellen gibt es mehrere Lesarten des hebräischen Textes. Dementsprechend unterschiedlich sind auch die Übersetzungen. Am Anfang des Psalms steht ein Aufschrei zu Gott, ausgelöst durch eine Situation der Not und Bedrängnis, gefolgt von einer Klage, die in viele Lebenssituationen passt:

2 Gib meinem Gebet, Gott, ein Ohr!

Verbirg dich nicht vor meinem Flehen!

3 Hör mir zu! Antworte mir (ענה 'anāh)!

Ich irre umher in meiner Verzweiflung, bin verwirrt - .

4 Die Gewalttäter schreien laut, die Unrecht tun, bedrängen mich,

Unheil lassen sie auf mich herabfallen, beschuldigen mich wutschnaubend.

5 Mein Herz bebt in meiner Mitte (בקרבי b qirbî), Todesschrecken sind auf mich gefallen, 6 Angst und Zittern kommen zu mir, Schrecken bedecken mich. ${ }^{43}$

Ps 55,2-6 ist eine umfassende Schilderung von Not, der klassische Beginn eines Klagepsalms. Eine Lektüremöglichkeit ist, diesen Psalm als Gebet einer vergewaltigten Frau zu lesen, wie das Ulrike Bail vorgeschlagen hat. ${ }^{44}$ Auch wenn damit nichts über historische Zuordnungen gesagt wird und die Frage, wie weit Frauen in den offiziellen Tempelkult in Israel einbezogen waren, letztlich offen bleiben

40 Vgl. Gunkel / Begrich 1985, S. 214 u.a.

41 Vgl. Kraus 1989, S. 561.

42 Ruwe 2016, S. 186.

43 Die Übersetzungen von Psalm 55 werden nach Bail 2006 zitiert.

44 Vgl. Bail 1994; dies. 1998. 
muss, ermöglicht das Konzept der „female voices“, Frauenstimmen als eine mögliche Leseperspektive sichtbar zu machen. Es geht dabei nicht um historische Rekonstruktion oder die Frage der Verfasserschaft von Frauen, sondern um die Frage, ,[...] ob Ps 55 den spezifischen Gewalterfahrungen von Frauen Raum geben kann und ob der Psalm die abwesenden und ins Schweigen verbannten Stimmen Tamars [und Dinas aus den oben genannten Erzählungen, Anm. der Autorinnen] [...] hörbar anwesend vergegenwärtigen kann. “45 Gewalterfahrungen werden vom Rand in die Mitte geholt, thematisiert und ins Gebet gebracht.

Das „Umherirren“ in V. $3^{46}$ und die Notschilderung in V. 5-6-das Beben des Herzens, Todesschrecken, Angst, Zittern, Schrecken - könnte „die psychischen Folgen einer Vergewaltigung wie depressive Hoffnungslosigkeit, mangelndes Selbstwertgefühl und Beschädigung der Identität " ${ }^{\text {47 }}$ umfassen. Diese Not wird in V.3 mit der Aufforderung „Antworte mir!“, in derselben Wurzel ענה 'anah, die wir in den Erzählungen und Gesetzestexten (in einer anderen Stammform) mit der Bedeutung „demütigen, vergewaltigen“ kennen gelernt haben, als Appell an Gott gerichtet.

Während die Feinde und Gewalttäter (V.4) in den Psalmen meistens als äußere Feinde oder politische Gegner verstanden werden, besteht eine Interpretationsmöglichkeit auch darin, sie als Feinde in der Nähe der betenden Person zu sehen. ${ }^{48}$ Das Motiv, dass frühere Freunde zu Feinden werden, ist auch sonst weit verbreitet (vgl. z. B. Klgl 1,2).

\section{Die Stadt als Raum der Gewalt (V. 10b-12):}

Ich sehe rohe Gewalt und Streit in der Stadt.

11 Sie umkreisen sie tags und nachts auf ihren Mauern.

Unheil und Leid in ihrer Mitte (בקרבה b eirbāh).

12 Verwüstung in ihrer Mitte (בקרבה be $b^{\mathrm{e}}$ qirbāh).

Von ihrem Markt weichen Unterdrückung und Betrug nicht.

V. 10b-12 zeichnen das Bild einer Stadt als Ort der Gewalt: Sowohl die Stadt als auch die Gewalt werden personifiziert dargestellt: Im Inneren der Stadt / in ihrer Mitte / in ihren Eingeweiden ist Gewalt: Das Innere (קרב qæræb) der betenden Person, das nach V. 5 in Aufruhr, Angst und Schrecken ist, wird in V.11.12 mit dem Inneren der Stadt parallel gesetzt, in dem Unheil, Leid und Verwüstung sind. Auch die Missstände treten personifiziert auf: Gewalt und Streit umkreisen die Stadt (V. 10.11), und nach V. 12 weichen Unterdrückung und Betrug nicht vom Markt der Stadt. Hier finden sich Begriffe von allgemeinen Missständen, ohne dass konkrete Gewalttaten genannt werden. Die Stadt, die eigentlich im Alten

\footnotetext{
45 Bail 1998, S. 160.

46 Vgl. Barré 2001.

47 Ebd., S. 173,

48 Vgl. Bail 1998, S. 160.
} 
Orient Ort des Schutzes ist, wird hier umgekehrt als Ort von Gewalt und Schutzlosigkeit dargestellt.

\section{Die Wüste als Gegenraum - Überleben im Bild der Taube (V. 7-9)}

Die räumlichen Zuordnungen sind in Ps 55 umgekehrt: Die Stadt, sonst Ort des Schutzes und der Geborgenheit, wird hier als beengter, bedrohlicher Raum wahrgenommen. Die Wüste, sonst Ort des Todes und der Gefahr, wird hier als Gegenraum zur Stadt, als Bereich der Freiheit entworfen, sie wird ,zum Asyl für Ausgestoßene und Flüchtlinge, “49 zum Zufluchtsort. Auch Hagar, Mose, David, Elia u. a. flüchten in die Wüste. Mit dem Symbol der Taube entwickelt der Beter oder die Beterin eine Fluchtphantasie, um aus der Gewalt zu entkommen:

${ }^{7}$ Ich spreche: Hätte ich Flügel gleich der Taube -

fliegen wollte ich und Ruhe finden.

${ }^{8}$ Weit, weit weg möchte ich flüchten, in der Wüste übernachten.

${ }^{9}$ zu meinem Zufluchtsort eilen, fort vom reißenden Wind, vom Sturm.

Die Symbolik der Taube ist vielschichtig: In der Erzählung von der großen Flut tut sie in der Situation der Not das, was die Menschen nicht können, sie fliegt voraus (Gen 8,8-12). Die Taube ist ein Opfertier, ein Ersatzopfer (Lev 12,6). In der Bildsprache des Hohen Liedes hat die Taube die Rolle einer Liebesbotin. Sie steht auch bildlich für die Geliebte (Cant 2,14; 5,2), oder auch für die Augen des/r Geliebten (Cant 1,15). Die Taube unterstreicht die Bedeutung der Wüste als „Gegenraum“.

Das Gebet ermöglicht, aus dem Verstummen herauszukommen, das sexuelle Gewalt auslöst. Mit der Fluchtphantasie beginnt eine posttraumatische Bearbeitung. Ulrike Bail deutet den Befund, dass die Verse 7-9 unverbunden im Kontext stehen, als sprachlichen Ausdruck von Dissoziation: Die Abspaltung des (psychischen) Ichs in die Utopie der Flucht ist eine Überlebensstrategie. ${ }^{50}$

\section{Der Täter - Missbrauch der Nähe (V. 14-15)}

Die sexuelle Gewalt gegen Frauen ist ein Aspekt von Gewalt, die in den Psalmen häufig den Feinden zugeordnet wird. Feinde sind in den Psalmen allgegenwärtig. Die Bedrohung, die von ihnen ausgeht, wird in vielfältigen Sprachbildern aus unterschiedlichen Bereichen drastisch geschildert: Vergleiche aus der Pflanzenund Tierwelt stehen neben Kampf- und Kriegsrhetorik. Die Sprache wird körperlich konkret, indem menschliche Körperteile genannt werden. Die Psalmen enthalten viele Appelle an Gott, den Beter oder die Beterin im Kampf gegen die Feinde zu unterstützen. „Die ,Feindpsalmen“ sind ein Weg, den aggressiven

49 Talmon 1984, S. 678.

50 Vgl. Bail 1998, S. 170. 
Feindbildern ihre Destruktivität zu nehmen und sie in konstruktive Kraft umzuwandeln. “51

Diese Bedrohung von außen, die in Ps 55 auch präsent ist, bekommt in V. 14-15 und 20-22 eine weitere Facette, indem hier die Bedrohung von einer bisher vertrauten Person in der Nähe ausgeht:

14 Du aber - ein Mensch meinesgleichen, mein Freund, mein Vertrauter, 15 die wir miteinander die Gemeinschaft (דô sôd) süß machten, im Haus Gottes wandelten in der Menge.

20 Sie kennen keine Verpflichtung, fürchten Gott nicht.

21 Sie erheben ihre Hände gegen ihre Vertrauten, brechen die gegenseitige Verpflichtung.

22 Glatter als Butter schmeichelt ihr Mund, aber handfester Streit liegt ihnen am Herzen.

Weicher als Öl fließen ihre Worte, aber sie sind offene Messer.

Die Formulierungen von Ps 55,14-15 (,ein Mensch meinesgleichen, mein Freund, mein Vertrauter") lassen sich mit dem Befund in Verbindung bringen, dass Vergewaltigung häufig im Kreis der Familie oder enger Vertrauter geschieht: Das „Erkennen“ (ידע yd'), das in „mein Vertrauter / Bekannter“ in V. 14 enthalten ist, hat in der Hebräischen Bibel häufig sexuelle Konnotationen (s. o. 2.1.). Auch in dem mehrdeutigen V. $15^{52}$ klingt in den Formulierungen „süß machen“ und סוד sôd („Gemeinschaft“ oder „Geheimnis“) ein Vertrauensverhältnis an.

Ulrike Bail bringt diese Topographie der Nähe mit gegenwärtigen Beobachtungen zusammen: „Etwa die Hälfte aller Vergewaltigungen wird von Männern verübt, die die Frau vor der Tat schon kennt. Zwei Drittel der Vergewaltigungen finden nicht im Freien, sondern im Haus statt. “53

\section{Hoffnung auf Rettung durch Gott}

Am Ende des Psalms steht die Hoffnung auf Unterstützung durch Gott, der sich für Menschen in Bedrängnis einsetzt. Am Schluss findet die betende Person trotz aller Sprachlosigkeit zu eigener Identität in der Formelsprache der Klagepsalmen: „Ich aber, ich vertraue auf dich“ (V. 24). Auch V. 17.18b.20 formulieren die Hoffnung auf Befreiung. Die Hoffnung, dass Gott hört, antwortet oder demütigt, wird in die Hand Gottes gelegt. Hier wird ein Bild von einem Gott gezeichnet, der auf der Seite der Bedrängten, Benachteiligten und Verfolgten steht. Der Gott Israels ist ein leiden-schaftlicher Gott: Er leidet mit, wo Menschen Feindschaft,

51 Zenger 2003, S. 7.

52 Vgl. die Übersetzung von Kraus 1989, S. 559: „die wir doch zusammen die Wonne der Gemeinschaft erlebten."

53 Bail 1996, S. 173. 
Bedrohung und Gewalt erleben und setzt sich leidenschaftlich, vehement und mit ganzer Kraft für sie ein. Er ist ein Gott, der Gewalt als Realität ernst nimmt und sich gleichzeitig für ihr Ende einsetzt.

Mit dieser Auslegung ist nichts über den realen historischen Kontext von Ps 55 ausgesagt. Aber die gender-sensible Zugangsweise ermöglicht es, nach „female voices" zu suchen und Erfahrungen zur Sprache zu bringen, die sprachlos machen.

Transformation ist also einerseits auf der Textebene angedeutet und andererseits in der Lektüre möglich. ,Trotz der historischen Distanz lädt die Offenheit der Bildersprache der Psalmen, die schon im Laufe der jüdischen und christlichen Auslegungsgeschichte immer wieder neu gedeutet wurde, dazu ein, Erfahrungen von Krieg und Gewalt zur Sprache zu bringen, diese Texte sozusagen als sprachliche Ventile zu nutzen. ${ }^{654}$ Psalmen sind Gebete, an Gott adressierte Hilferufe. Die elementare Sprache der Psalmen ermöglicht es, Gewalt wahrzunehmen und Erfahrungen auszusprechen. „Die Feindpsalmen leben vor allem von der Spannung der in ihnen gestalteten destruktiven und konstruktiven Bilder. In ihnen sind Bilder der Angst und Bilder der Hoffnung so intensiv miteinander verwoben, dass das Hineintauchen in diese Bildwelt geradezu therapeutische Kräfte in uns freisetzen kann. ${ }^{\text {55 }}$ Die Psalmen machen auf gesellschaftliche Missstände aufmerksam. In ihrer vielfältigen Bildersprache haben sie das Potential, Transformationsprozesse anzuregen.

\subsection{Klagelied 1,8-10}

Als zweites Beispiel für sexuelle Gewalt gegen Frauen in einem poetischen Text soll hier ein kurzer Abschnitt aus den Klageliedern gelesen werden. Es ist wohl davon auszugehen, dass es sich um fünf eigenständige anonyme Lieder handelt, die im Laufe des 6. Jahrhunderts, in Reaktion auf die Zerstörung Jerusalems durch die Babylonier 587 v. Chr. in Jerusalem entstanden sind. ${ }^{56}$ Das 1 . Klagelied schildert die Katastrophe aus der Perspektive unmittelbarer Betroffenheit, die literarische Stilisierung deutet aber doch auf einen gewissen zeitlichen Abstand hin. Allerdings ist von Rückkehrbestrebungen noch nicht die Rede, sodass Klgl 1 ungefähr in den Jahren 580-550 entstanden sein dürfte. ${ }^{57}$ Das Lied ist formal als Akrostichon gestaltet, die einzelnen Verse beginnen jeweils mit einem Buchsta-

54 Grohmann 2015, S. 46.

55 Zenger 2003, S. 149.

56 Gegen eine zu frühe Ansetzung spricht sich Frevel 2002, S. 103, aus: Als literarisch konstruierte Texte müssen sie nicht unmittelbar nach der Zerstörung Jerusalems verfasst worden sein. Frevel sieht Lied 2 als das älteste an, Klgl 4 datiert er auf spätexilische bis frühnachexilische Zeit.

57 Vgl. Koenen 2014, S. 30. 
Marianne Grohmann / Agnethe Siquans

ben des Alphabets. Das deutet darauf hin, dass es von Anfang an als schriftlicher Text konzipiert war. Das Lied enthält Elemente der altorientalischen Stadtuntergangsklage, der Toten- oder Leichenklage (vgl. 2 Sam 1,19-27; 3,33 f.) ${ }^{58}$, der Klagelieder eines/einer einzelnen sowie der Volksklagelieder. Es ist aus diesen Elementen aber auf unterschiedliche Weise zu einer eigenen literarischen Kreation zusammengestellt worden.

In den Klageliedern wird die ganze Stadt Jerusalem personifiziert als Frau in unterschiedlichen Rollen dargestellt. Das Trauma der Zerstörung Jerusalems durch die Babylonier im 6. Jahrhundert v. Chr. wird in allen fünf Liedern oder Gebeten verarbeitet. Die Gewalt, die das Volk Israel erfahren hat, wird drastisch geschildert, nicht nur, aber auch als sexuelle Gewalt gegen eine Frau. Transformationsperspektiven sind hier wenig im Blick.

\section{Klgl 1,8-10}

8 Verfehlt, verfehlt hat sich Jerusalem. Darum ist sie zum Gespött geworden.

Alle ihre Verehrer verachten sie, denn sie haben ihre Blöße (ערוה 'ærvāh) gesehen. Und sie: Sie seufzt und hat sich abgewendet.

9 Ihre Unreinheit ist an ihren Säumen, sie denkt nicht an ihre Zukunft.

Sie ist schrecklich gefallen. Niemand ist da, sie zu trösten.

„Sieh, JHWH, mein Elend (ענה 'ônî) an, denn der Feind ist groß geworden!“

10 Seine Hand hat der Bedränger nach allen ihren Schätzen ausgestreckt.

Sie hat gesehen, wie Völker in ihr Heiligtum kamen,

wo du geboten hast, nicht in deine Versammlung zu kommen. ${ }^{59}$

Im 1. Teil des Liedes (V. 1-11) schildert eine externe Stimme die Zustände in Jerusalem. Die Stadt tritt in personifizierter Form als einsame Witwe, versklavte Fürstin, Klagefrau, verlassene Geliebte, Jungfrau und ihrer Kinder beraubte Mutter auf. In V. 9c und 11c sind Stimmen der Stadt eingeflochten, mit „Sieh, JHWH!“ eingeleitete Appelle an Gott.

In V. 8 taucht das Motiv der Verachtung durch frühere Vertraute / Verehrer wieder auf. Mit dem „Sehen der Blöße“" klingen Tora-Bestimmungen an, wie z.B. aus Lev 18, wo es um unerlaubte sexuelle Kontakte, v. a. Inzest geht: diese werden jeweils mit dem Ausdruck „die Blöße aufdecken“ beschrieben (גלה ערוה glh 'ærvāh). Die „Blöße / Scham“ kann auf Männer und Frauen bezogen werden: So ist z.B. in Lev 20,17 „die Blöße sehen“ wechselseitig beim Inzest mit der Schwester angesprochen. Nach Gen 9,22 sieht Ham, der Stammvater Kanaans "die Blöße seines Vaters“, des betrunkenen Noah. In übertragenem Sinn ist in Gen 42,9.12 von der Blöße des Landes die Rede.

58 Vgl. Jahnow 1923.

59 Deutsche Übersetzung MG. 
„Sie (die Stadt Jerusalem) seufzt und wendet sich ab“ (V. 9) lässt sich als Reaktion der Scham, Trauer und Klage der personifizierten Stadt deuten. Die im selben Vers angesprochene Unreinheit - der Zustand der Kultunfähigkeit, ein Zustand, in dem man sich nicht dem Heiligen nähern, nicht ins Heiligtum kommen soll (V. 10) - ist ein in seiner Wirkungsgeschichte gerade unter GenderPerspektive höchst problematischer Begriff. Mit der „Unreinheit an den Rändern / an den Säumen" wird hier nicht gesagt, wodurch die Unreinheit hervorgerufen wird: entweder durch illegitimen Geschlechtsverkehr / Ehebruch oder durch Blut - Menstruationsblut oder durch Vergewaltigung? „Jerusalem denkt / erinnert sich (זכר zkr) nicht an ihre Zukunft / ihr Ende / wie es mit ihr enden könnte“ (V. 9): Hier begegnet uns wieder die Wurzel ענה 'ônî (Elend), die auch für Vergewaltigung verwendet wird: Das umfassende Elend, die Not, von der Vergewaltigung ein Teil ist, wird in Gebetssprache gefasst und als Hilferuf an Gott gerichtet. Auch dieser Text enthält vielfältiges Feindvokabular: Die Feinde sind „Bedränger“ / „die, die das Leben eng machen“ (V. 10: צsar).

Klagelied 1 enthält wenige Hoffnungsperspektiven. Aber zumindest wird die Not umfassend in ihren verschiedenen Facetten gesehen und benannt. Sexuelle Gewalt wird nicht tabuisiert, sondern in sprachlichen Bildern beschrieben: In dem Satz „Seine Hand hat der Bedränger nach allen ihren Schätzen ausgestreckt" (V. 10) klingen einerseits die Tempelschätze Jerusalems als Stadt an und andererseits die Schönheit / das Begehrenswerte Jerusalems als Frau.

Neben diesen Umschreibungen wird an einer anderen Stelle, in Klgl 5,11, sexuelle Gewalt explizit ausgesprochen: „Frauen haben sie in Zion vergewaltigt (ענה pi. 'innāh), Jungfrauen in den Städten Judas." Im Kontext einer gemeinsamen Klage, mit der Gott zum „Gedenken“ aufgefordert wird, wird Vergewaltigung als eine von vielen Nöten in der Stadt aufgezählt. „Dabei wird sexuelle Gewalt gegen Frauen als Kriegswaffe sichtbar. Diese Gewalt basiert auf einer doppelten Differenz, der Differenz von Eroberern und Eroberten und der Differenz von Männern und Frauen.“60

Sexuelle Gewalt wird in den Klageliedern insofern transformiert, als sie von einer bloßen Beschreibung der Zustände und der Erfahrungen von Gewalt in einen Hilferuf, einen Appell an Gott verwandelt wird: „Sieh, Gott, mein Elend / meine Bedrängnis / Not / die Gewalt, die ich erfahren habe“ (V. 9). Die Texte geben Erfahrungen, die sprachlos machen, einen Ausdruck.

60 Häusl 1999, S. 276. 
Marianne Grohmann / Agnethe Siquans

\section{Hermeneutische Perspektiven zum Umgang mit Gewalttexten in der Hebräischen Bibel - Übersetzung und Interpretation als Transformationsprozesse}

Texte in der Hebräischen Bibel, die mit sexueller Gewalt zu tun haben, sind häufig „texts of terror"661. Das Schockierende dieser Texte steht in Widerspruch zu ihrem Anspruch als kanonische Texte. Ilse Müllner schlägt vier Fragerichtungen vor, um sich diesen Texten anzunähern:

1. Am Anfang steht die Frage danach, „ob die Bibel so etwas wie einen Begriff von sexueller Gewalt hat und ob sich diese Definition von gegenwärtigen Vorstellungen von sexueller Gewalt unterscheidet"62. - Die genannten Textbeispiele zeigen, dass ein Unrechtsbewusstsein da ist, das sich aber im Rahmen der altorientalischen patriarchalen Gesellschaften bewegt und sich daher in jedem Fall von gegenwärtigen Diskursen unterscheidet. Die Erzähltexte betrachten aus androzentrischer Perspektive sexuelle Gewalt an Frauen deshalb als Problem, weil sie die Rechte anderer Männer und damit die gesellschaftliche Ordnung gefährdet.

2. „Die biblischen Texte müssen daraufhin befragt werden, inwieweit sie sexuelle Gewalt [...] zum Thema machen." ${ }^{63}$ - Sie tun das einerseits konstatierend, indem sie Gewalt als einen Teil menschlicher Realität feststellen, und andererseits in poetischer, zum Teil bildlicher Sprache: Die narrativen Texte, besonders die Erzählungen über Dina, die Frau des Leviten und Tamar, sprechen sexuelle Gewalt direkt und unverblümt an. Sie bewerten diese auch deutlich als unerwünscht (,Schandtat“). In den Psalmen lassen Menschen in Not ihren Ängsten, ihrer Klage und ihren Phantasien freien Lauf. Die dramatischen Schilderungen von Gewalt machen die Bearbeitung von Ängsten in einer Bedrohungssituation überhaupt erst möglich, indem sie verbalisiert werden. Die Verbalisierung und Verschriftlichung dieser Erfahrungen nötigen zu einer Auseinandersetzung. Verdrängung und Leugnung der Realität von sexueller Gewalt sind so nicht mehr möglich. Damit eröffnet sich ein erster Weg zur Veränderung.

3. Wichtig ist die Frage nach der Wertung in den Texten: „Nicht jeder Text, der Gewalt thematisiert, befürwortet sie.“64 - Gerade die Erzählungen über Dina und Tamar machen deutlich, dass sich Erzählungen durch ihre Darstellungsweise auch von der dargestellten Gewalt distanzieren. „So etwas tut man nicht in Israel“" (2 Sam 13,12), lässt die Erzählung Tamar selbst urteilen (vgl. auch Gen 34,7; Dtn 22,21). Sexuelle Gewalt wird als gesellschaftlich unerwünschtes

61 Trible 1984.

62 Müllner 1999, S. 43.

63 Ebd.

64 Ebd. 
Verhalten qualifiziert. Außerdem wird Gott davon distanziert. Gewalt wird häufig als menschliches Phänomen gezeichnet, dem Gott fern steht.

4. „Jeder Text ist mit dem und gegen den Strich auszulegen. " ${ }^{65}$ Spiegelt der Text reale gesellschaftliche Verhältnisse? Texte können in Bezug auf Gender-Fragen ganz unterschiedliches Potential enthalten, wie z.B. Ps 55 in der vorgestellten Interpretation von Ulrike Bail. Die Erzählungen über sexuelle Gewalt zeigen, dass sich auch hier die Perspektiven verschieben können (,reversed rape“): Die Schilderung einer Vergewaltigung kann umgekehrte Verhältnisse darstellen. Diese Texte halten damit den Blick auf eine andere Deutung offen, so wie es auch die „Erinnerungstexte“ tun. Die Möglichkeit zur Veränderung von Realität eröffnet sich mit dem Erzählen, dem Erinnern und einer Offenheit der Texte, die eine Interpretation „gegen den Strich“ zulässt.

Darüber hinaus ist die historische Einordnung wichtig: Die biblischen Texte sind in ihrem Kernbestand vor über 2500 Jahren im alten Vorderen Orient entstanden. Meistens haben sie einen langen Weg von Überarbeitungen und damit Transformationen hinter sich, der sich in unterschiedlichen Übersetzungen und Auslegungen fortsetzt. In der Exilszeit (6. Jahrhundert v. Chr.), einer wesentlichen Transformationsphase der biblischen Texte, war das Volk Israel eine kleine, machtlose Gruppe. Die Gewalterfahrungen werden von Menschen formuliert, die sich in einer Situation realer und konkreter Bedrängnis befinden. Es sind Hilfeschreie von Machtlosen. Es ist wichtig, sich den historischen Abstand immer wieder vor Augen zu führen. Nicht alles, was im alten Israel passend war, lässt sich 1:1 auf Europa am Beginn des 21. Jahrhunderts übertragen. Es macht Transformationen in der Interpretation notwendig.

Es bleibt eine wichtige Aufgabe der Exegese, die biblischen Texte möglichst genau in ihre historischen und literarischen Kontexte einzuordnen und gleichzeitig nach ihrer Relevanz zu fragen.

Die kanonische Autorität der Bibel bedeutet nicht, dass ChristInnen dem Inhalt des Geschriebenen ungebrochen zustimmen müssten, sondern dass ihnen diese Bücher zum Lesen und Auslegen aufgegeben sind. Die Auslegung der Schrift - nicht die Unterwerfung unter einzelne Inhalte - bildet eine der Grundlagen christlicher Identität. ${ }^{66}$

Das weit verbreitete Image von „Sex and Crime“ muss sich die Hebräische Bibel zum Teil gefallen lassen. Aber sie hält den Menschen im alten Israel genauso wie uns heute einen Spiegel vor. Gewalt wird als Teil menschlicher Erfahrungen zur Sprache gebracht. Schonungslos werden Leserinnen und Leser mit den schmerzhaften Fakten konfrontiert. Erzählt werden Handlungen und Taten. Gefühle drücken sich oft implizit in sprachlichen Bildern aus. Gewalt wird als

65 Ebd.

66 Ebd., S. 42. 
Marianne Grohmann / Agnethe Siquans

menschliche Realität dargestellt, Gott steht in den Erzählungen häufig im Hintergrund. Manchmal verurteilt der biblische Text selbst diese Gewalt und prangert sie - z. B. in der Richterzeit - als Zeichen von Chaos und Missständen an. In Gebetstexten wird Gott mit der Hoffnung angerufen, der Gewalt ein Ende zu setzen.

Die Verbalisierung sexueller Gewalt in biblischen Texten beinhaltet aber bereits Wege der Transformation. In unterschiedlicher Weise zeigt sich in den Texten selbst eine veränderte Wahrnehmung und Bewertung von Gewalt, besonders deutlich in den Gesetzestexten, wo ein Textvergleich die veränderte Beurteilung und die unterschiedlichen Konsequenzen sichtbar macht. In der narrativen Darstellung sexueller bzw. sexuell konnotierter Gewalt wird insbesondere das Geschlechterverhältnis transformiert, allerdings mit dem Ziel, die soziale Ordnung aufrecht zu erhalten. In den poetischen Texten ist eine Transformation von Gewalterfahrungen zu beobachten, die ebenfalls neue Bewertungen und damit Zukunftsperspektiven eröffnet. Transformationsprozesse finden einerseits auf der Ebene der biblischen Texte selbst statt, lassen sich andererseits aber auch auf der Lektüreebene initiieren.

\section{Literaturverzeichnis}

Abasili, Alexander Izuchukwu: „Was It Rape? The David and Batsheba Pericope Reexamined“, in: $V T$ (61) 2011, S. 1-15.

Bail, Ulrike: „,Vernimm Gott, mein Gebet. Psalm 55 und Gewalt gegen Frauen“, in: Jahnow, Hedwig u.a. (Hg.): Feministische Hermeneutik und Erstes Testament. Analysen und Interpretationen. Stuttgart u. a. 1994, S. 67-84.

Bail, Ulrike: Gegen das Schweigen klagen. Eine intertextuelle Studie zu den Klagepsalmen Ps 6 und Ps 55 und der Erzählung von der Vergewaltigung Tamars. Gütersloh 1998.

Bail, Ulrike u. a. (Hg.): Bibel in gerechter Sprache. Gütersloh 2006.

Bal, Mieke: Murder and Difference. Gender, Genre, and Scholarship on Sisera's Death. Bloomington / Indianapolis 1988.

Barré, Michael L.: ,,Wandering about' as a Topos of Depression in Ancient Near Eastern Literature and in the Bible“, in: JNES (60) 2001, S. 177-187.

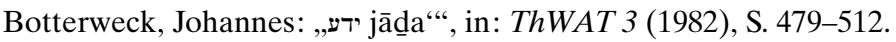

Brenner, Athalya: „M Text Authority in Biblical Love Lyrics: The Case of Qohelet 3.1-9 and its Textual Relatives“, in: Brenner, Athalya / Dijk-Hemmes, Fokkelien van: On Gendering Texts. Female and Male Voices in the Hebrew Bible. Leiden 1993, S. $133-$ 163.

Dietrich, Walter / Link, Christian: Die dunklen Seiten Gottes. Neukirchen-Vluyn 1995.

Eder, Sigrid: „Gewalt in der Bibel. Begrifflichkeit - Verstehenshilfen - Perspektiven“, in: $P Z B$ (19) 2010, S. 1-20.

Engelken, Karen: פלגש pilæḡæš“, in: ThWAT VI (1989) S. 586-589. 
Exum, J. Cheryl: „Das Buch der Richter. Verschlüsselte Botschaften für Frauen“, in: Schottroff, Luise / Wacker, Marie-Theres (Hg.): Kompendium Feministische Bibelauslegung. Göttingen ${ }^{2} 1999$, S. 90-103.

Fewell, Danna Nolan / Gunn, David M.: „Controlling Perspectives. Women, Men and the Authority of Violence in Judges 4 \& 5“, in: JAAR (58) 1990, S. 389-411.

Finsterbusch, Karin: „Frauen zwischen Fremdbestimmung und Eigenständigkeit. Genderrelevantes in den Gesetzestexten der Tora“, in: Fischer, Irmtraud et al. (Hg.): Tora (Die Bibel und die Frauen. Hebräische Bibel - Altes Testament 1.1). Stuttgart 2010, S. $375-400$.

Fokkelman, Jan P.: Narrative Art and Poetry in the Books of Samuel. A Full Interpretation Based on Stylistic and Structural Analyses. Vol. 1 King David (II Sam. 9-20 \& I Kings 1-2). Assen 1981.

Frevel, Christian: „Zerbrochene Zier. Tempel und Tempelzerstörung in den Klageliedern (Threni)“, in: Keel, Othmar / Zenger, Erich (Hg.): Gottesstadt und Gottesgarten. $\mathrm{Zu}$ Geschichte und Theologie des Jerusalemer Tempels (QD 191). Freiburg u. a. 2002, S. 99-153.

Grohmann, Marianne: „Ein Gott der Rache? Feindpsalmen in jüdischen und christlichen Auslegungen“, in: Rossmanith, Nadja u. a. (Hg.): Sprachen heiliger Schriften und ihre Auslegung. Wien 2015, S. 35-48.

Groß, Walter: Richter (Herders Theologischer Kommentar zum Alten Testament). Freiburg 2009.

Gunkel, Hermann / Begrich, Joachim: Einleitung in die Psalmen. Die Gattungen der religiösen Lyrik Israels. Göttingen ${ }^{4} 1985$.

Häusl, Maria: „Die Klagelieder. Zions Stimme in der Not“, in: Schottroff, Luise / Wacker, Marie-Theres (Hg.): Kompendium Feministische Bibelauslegung. Gütersloh ${ }^{2} 1999$, S. 270-277.

Hentschel, Georg: „Das Buch der Richter“, in: Zenger, Erich u.a. (Hg.): Einleitung in das Alte Testament, hg. von Christian Frevel. Stuttgart ${ }^{9} 2016$, S. 267-277.

Jahnow, Hedwig: Das hebräische Leichenlied im Rahmen der Völkerdichtung (BZAW 36). Gießen 1923.

Jost, Renate / Seifert, Elke: „Das Buch Ezechiel. Männerprophetie mit weiblichen Bildern“, in: Schottroff, Luise / Wacker, Marie-Theres (Hg.): Kompendium Feministische Bibelauslegung. Gütersloh ${ }^{2} 1999$, S. 278-290.

Keefe, Alice: „Rapes of Women / Wars of Men“, in: Camp, Claudia V. / Fontaine, Carole R. (Hg.): Women, War, and Metaphor. Language and Society in the Study on the Hebrew Bible (Semeia 61). Atlanta, GA 1993, S. 79-97.

Knauf, Ernst Axel: Richter (Zürcher Bibelkommentare). Zürich 2016.

Koenen, Klaus: Klagelieder (Threni) (BKAT 20/1). Neukirchen-Vluyn 2014.

Kraus, Hans-Joachim: Psalm 1-59 (BKAT 15/1). Neukirchen-Vluyn ${ }^{6} 1989$.

Müllner, Ilse: Gewalt im Hause Davids. Die Erzählungen von Tamar und Amnon (2 Sam 13,1-22) (HBS 13). Freiburg im Breisgau 1997.

Müllner, Ilse: „Sexuelle Gewalt im Alten Testament“, in: Ulrike Eichler / Müllner, Ilse (Hg.): Sexuelle Gewalt gegen Mädchen und Frauen als Thema der feministischen Theologie. Gütersloh 1999, S. 40-75.

Paganini, Simone: „Gesetze für, gegen bzw. über Frauen im Buch Deuteronomium“, in: PZB (19) 2010, S. 21-34. 
Ruwe, Andreas: „Vertrauenszuwachs in der Klage. Zu Gliederung und Aussagegefälle von Psalm 55“, in: ders. (Hg.): Du aber bist es, ein Mensch meinesgleichen (Psalm 55,14). Ein Gespräch über Psalm 55 und seine Parallelen (BThSt 157). NeukirchenVluyn 2016, S. 147-189.

Scholz, Susanne: Sacred Witness. Rape in the Hebrew Bible. Minneapolis, Minn. 2010. Talmon, Shemaryahu: Art. מִדִ mid

Trible, Phylis: Texts of Terror. Literary Feminist Readings of Biblical Narratives. Philadelphia 1984.

Trible, Phylis: Mein, Gott, warum hast du mich vergessen! Frauenschicksale im Alten Testament. Gütersloh ${ }^{2} 1990$.

Van Wolde, Ellen: „Does 'innâ Denote Rape? A Semantic Analysis of a Controversial Word“, in: VT (52) 2002, S. 528-544.

Yamada, Frank M.: Configurations of Rape in the Hebrew Bible. A Literary Analysis of Three Rape Narratives (Studies in Biblical Literature 109). New York 2008.

Zenger, Erich: Ein Gott der Rache? Feindpsalmen verstehen (Psalmenauslegungen 4). Freiburg / Basel / Wien 2003.

Ao. Univ.-Prof. Mag. Dr. Agnethe Siquans, Institut für Bibelwissenschaft, KatholischTheologische Fakultät Universität Wien, Schenkenstraße 8-10, 1010 Wien, Austria, agnethe.siquans@univie.ac.at

Ao. Prof. Dr. Marianne Grohmann, Institut für Alttestamentliche Wissenschaft und Biblische Archäologie, Evangelisch-Theologische Fakultät der Universität Wien, Schenkenstraße 8-10, 1010 Wien, Austria, marianne.grohmann@univie.ac.at

Citation: Grohmann, Marianne / Siquans, Agnethe: „Literarische Transformationen sexueller Gewalt in der Hebräischen Bibel“, in: Heller, Birgit (ed.): Religion, Transformation and Gender. (J-RaT 2017 / 2), pp. 157-184.

Datum der Publikation: 02.10.2017 